Research Article

\title{
Molecular Detection of Antibiotic-Resistant Genes in Pseudomonas aeruginosa from Nonclinical Environment: Public Health Implications in Mthatha, Eastern Cape Province, South Africa
}

\author{
Mojisola Clara Hosu $\mathbb{D}^{1},{ }^{1}$ Sandeep Vasaikar $\mathbb{D}^{1,2}$ Grace Emily Okuthe $\mathbb{D}^{\mathbb{D}}{ }^{3}$ \\ and Teke Apalata $\mathbb{D}^{1,2}$ \\ ${ }^{1}$ Division of Medical Microbiology, Department of Laboratory Medicine and Pathology, Faculty of Health Sciences, \\ Walter Sisulu University, Private Bag: X1, Mthatha 5117, Eastern Cape, South Africa \\ ${ }^{2}$ National Health Laboratory Services (NHLS), Nelson Mandela Academic Hospital, Mthatha 5100, South Africa \\ ${ }^{3}$ Department of Biological and Environmental Sciences, Walter Sisulu University, Private Bag, X1, Mthatha 5117, \\ Eastern Cape, South Africa
}

Correspondence should be addressed to Teke Apalata; ruffinapalata@gmail.com

Received 28 June 2020; Revised 25 October 2020; Accepted 26 December 2020; Published 5 January 2021

Academic Editor: Clemencia Chaves Lopez

Copyright (c) 2021 Mojisola Clara Hosu et al. This is an open access article distributed under the Creative Commons Attribution License, which permits unrestricted use, distribution, and reproduction in any medium, provided the original work is properly cited.

Evaluation of resistant profiles and detection of antimicrobial-resistant genes of bacterial pathogens in the nonclinical milieu is imperative to assess the probable risk of dissemination of resistant genes in the environment. This paper sought to identify antibiotic-resistant genes in Pseudomonas aeruginosa from nonclinical sources in Mthatha, Eastern Cape, and evaluate its public health implications. Samples collected from abattoir wastewater and aquatic environment were processed by membrane filtration and cultured on CHROMagarTM Pseudomonas medium. Species identification was performed by autoSCAN-4 (Dade Behring Inc., IL). Molecular characterization of the isolates was confirmed using real-time polymerase chain reaction (rPCR) and selected isolates were further screened for the possibility of harboring antimicrobial resistance genes. Fifty-one Pseudomonas species were recovered from abattoir wastewater and surface water samples, out of which thirty-six strains were Pseudomonas aeruginosa (70.6\%). The P. aeruginosa isolates demonstrated resistance to aztreonam (86.1\%), ceftazidime (63.9\%), piperacillin (58.3\%), cefepime (55.6\%), imipenem (50\%), piperacillin/tazobactam (47.2\%), meropenem (41.7\%), and levofloxacin (30.6\%). Twenty out of thirty-six P. aeruginosa displayed multidrug resistance profiles and were classified as multidrug-resistant (MDR) (55.6\%). Most of the bacterial isolates exhibited a high Multiple Antibiotic Resistance (MAR) Index ranging from 0.08 to 0.69 with a mean MAR index of 0.38 . In the rPCR analysis of fifteen P. aeruginosa isolates, 14 isolates (93.3\%) were detected harboring bla $a_{\mathrm{SHV}}$, six isolates (40\%) harbored $b l a_{\mathrm{TEM}}$, and three isolates $(20 \%)$ harbored $b l a_{\mathrm{CTX}-\mathrm{M}}$, being the least occurring ESBL. Results of the current study revealed that $P$. aeruginosa isolates recovered from nonclinical milieu are resistant to frontline clinically relevant antipseudomonal drugs. This is concerning as it poses a risk to the environment and constitutes a public health threat. Given the public health relevance, the paper recommends monitoring of multidrug-resistant pathogens in effluent environments.

\section{Introduction}

Antimicrobial resistance (AMR) is a public health crisis in both human and veterinary medicine $[1,2]$. The irrational use of antibiotics in both human medicine and animal production for growth-promoting purposes, metaphylaxis, and prophylaxis has fueled the proliferation and spread of antibiotic-resistant bacteria and resistance genes resulting in aggravated public health and environmental risks [3-5]. The threat posed by AMR to human health is particularly concerning in low- to middle-income countries (LMICs). This is due to the higher possibility of community-acquired 
resistant infections, the high transmissible disease burden in the general populace, and poor access to health services [6], thereby leading to increased morbidity, prolonged hospitalization, and increased healthcare costs, thus exerting an economic burden on family units and the society [7].

Pseudomonas aeruginosa ( $P$. aeruginosa), an environmental bacterium, can be found in various terrestrial and aquatic habitats. This is due to its extensive metabolic versatility that enhances its distribution, proliferation, and survival despite adverse physical and chemical conditions, thereby enhancing its ecological success and potential threat to public health $[8,9]$. $P$. aeruginosa is an opportunistic human pathogen competent for a wide array of infections including respiratory tract, blood, urinary tract, and skin infections [10]. This competence for infections and ability for antibiotic resistance has made the organism to be recognized as a threat to public health $[9,10]$. P. aeruginosa make use of both intrinsic and acquired resistance mechanisms. The acquired resistance mechanism is facilitated by mobile genetic elements through horizontal gene transfer (HGT). This poses a greater risk to human health because of the ease of expression and dissemination [11].

Antibiotic usage in the agricultural sector has compounded the spread of resistance in the human community due to the environmental dissemination of transferable resistance genes [12]. Abattoir wastes have the ability to contaminate both surface and groundwater. Discharge from abattoir effluent contaminates the environment by introducing pathogens that can affect land and water qualities, thus endangering human, animal, and aquatic ecosystem's health and constituting a menace to human health and environmental safety [13]. The possibility of pathogens from abattoir effluent and animal waste reaching or discharging into water bodies and developing resistance to antibiotics in human infection is a concern. This is because these infections are usually difficult to treat and often result in morbidity and mortality especially in the most vulnerable members of the community [14].

Several studies have investigated the prevalence and detection of extended-spectrum $\beta$-lactamase- (ESBL-) and metallo $\beta$-lactamase- (MBL-) producing $P$. aeruginosa isolated from clinical samples $[15,16]$, but there is a scarcity of data on the occurrence in nonclinical samples. The need to identify and monitor antibiotic-resistant genes in water bodies and wastewater is necessary to assess their potential risk to human health. The present study investigated the prevalence of antimicrobial resistance and antibiotic-resistant genes in nonclinical strains of Pseudomonas aeruginosa in Mthatha, Eastern Cape, and highlighted its public health implications.

\section{Materials and Methods}

2.1. Study Design and Setting. A cross-sectional study was conducted within the period of January to June 2019. The study sites were Umzikantu Red Meat Abattoir, Zimbane Mthatha, Mthatha River, and Mthatha Dam. Umzikantu Red Meat Abattoir is a high-throughput abattoir located in Zimbane location in Mthatha, Eastern Cape. It is the only operational red meat abattoir serving Mthatha and its environs in the OR Tambo District Municipality. It is certified and has the capacity to slaughter 50 units of animals on a daily basis. One unit equals one cow/ox or two calves or six sheep or four pigs. The abattoir is open to the public and offers slaughter and cutting services at an affordable price. It also doubles as a wholesaler supplying meat to butcheries, supermarkets, and hospitals.

Mthatha Dam $\left(31^{\circ} 33^{\prime} 2^{\prime \prime} \mathrm{S} 28^{\circ} 44^{\prime} 24^{\prime \prime} \mathrm{E}\right.$ Coordinates) is an earth-fill type dam on the Mthatha River, located close to Mthatha Town, in the OR Tambo District Municipality of the Eastern Cape. This dam was built in 1977 to serve municipal and industrial purposes. The Department of Water and Sanitation oversees the affairs of the dam. The catchment area of the dam is $886 \mathrm{~km}^{2}$ with a surface area measuring $25.42 \mathrm{~km}^{2}$. It has a height of $38 \mathrm{~m}$ with a length measuring $620 \mathrm{~m}$. The reservoir capacity of the dam is $253,674,000 \mathrm{~m}^{3}$.

2.2. Sample Collection. Abattoir wastewater samples: using standard methods for the examination of water and wastewater [17], $100 \mathrm{~mL}$ of abattoir effluents was taken from two sampling points into sterile bottles appropriately labeled. All samples were stored in cooler boxes for transportation to the Medical Microbiology laboratory at Walter Sisulu University Mthatha, for further analyses, within $4 \mathrm{~h}$ of sample collection.

Aquatic environment samples: water samples from the Mthatha Dam were collected aseptically in sterile $100 \mathrm{~mL}$ Duran Schott glass bottles from different sampling points by directly dipping the bottles about $20 \mathrm{~cm}$ below the surface of the water. After collection, the samples were stored in iced cooler boxes, transported to the laboratory, and kept at about $4^{\circ} \mathrm{C}$ until analyzed.

2.3. Bacteriological Analysis. The membrane filtration method was used for isolation according to standard methods [17]. For all samples, three volumes of $100 \mathrm{~mL}$ were filtered [10] through a $0.45 \mu \mathrm{m}$ pore sized gridded membrane filter (Whatman Laboratory Division, Maidstone, England) using a water pump (model Sartorius 16824). Filters were removed and aseptically placed on CHROMagarTM Pseudomonas (CHROMagarTM; Paris, France) agar plates ensuring that no air bubbles were trapped. All media were prepared according to the manufacturers' instructions (CHROMagarTM; Paris, France). Each sample was analyzed in triplicate. The plates were incubated aerobically at $37^{\circ} \mathrm{C}$ for 24-48 hours. Blue colonies which were characteristics of Pseudomonas spp. were subcultured to obtain pure cultures.

2.4. Characterization of Pseudomonas aeruginosa. Blue colonies typical of Pseudomonas species on the chromogenic medium were subcultured on both Cetrimide agar and CHROMagar to get pure colonies. The characteristic grapelike odor was a useful marker of identification. Phenotypic tests such as Gram stain, oxidase test, and catalase test were performed [18]. Species identification was carried out using 
Gram-negative ID type 2 panels (Beckman Coulter, Inc. USA) of MicroScan autoScan-4 automated system (Dade Behring Inc., Deerfield, IL). Growth at $42^{\circ} \mathrm{C}$ [18] in an aerobic incubator was also used to confirm the identity of the $P$. aeruginosa isolates. All of the strains were stored at $-80^{\circ} \mathrm{C}$ in $15 \%$ glycerol until further use.

2.5. Molecular Confirmation of Strains by rPCR. DNA Extraction: DNA was extracted from overnight colonies of a bacterial culture grown on Cetrimide agar. This was resuspended in Roche MagNA Pure Bacteria Lysis Buffer, vortexed briefly, heated at $95^{\circ} \mathrm{C}$ for 10 minutes, and pelleted by centrifugation at $13000 \mathrm{~g}$ for 10 minutes. Four hundred microliters were used as a specimen in the MagNA Pure Compact (MPC) System (Roche Applied Science, Indianapolis), using MPC Nucleic Acid isolation kit 1 according to the manufacturer's instructions. Elution tubes containing $200 \mu \mathrm{l}$ purified nucleic acids were stored at $-80^{\circ} \mathrm{C}$ until further use. The LightCycler 2.0 instrument (Roche Applied Science, Germany) and Fast start LightCycler 480 HybProbes Master Kit (Roche Diagnostics, USA) were used for polymerase reaction. Specific primers and probes (Table 1) designed by TIB Molbiol (Germany) targeting the gene, species-specific gyrB, were amplified by singleplex realtime polymerase chain reaction ( $\mathrm{rPCR}$ ) following the protocol shown in Table 2.

2.6. Antimicrobial Susceptibility Testing. Antimicrobial susceptibility testing was performed by MicroScan autoScan-4 system using dehydrated broth microdilution method in the MIC Panel Type 44 (NM44) (Beckman Coulter, Inc. USA) following the manufacturer's guidelines [20]. The following antibiotics were tested in the panels: amikacin, aztreonam, cefepime, ceftazidime, ciprofloxacin, doripenem, gentamicin, imipenem, levofloxacin, meropenem, piperacillin/tazobactam, piperacillin, and tobramycin. MICs were analyzed and interpreted according to the recommended clinical breakpoints given in CLSI guidelines [21]. ATCC Quality control organisms used were $P$. aeruginosa ATCC 27853 and Escherichia coli ATCC 25922. Nonsusceptibility includes a combination of resistance and intermediate resistance. Multidrug (MDR) $P$. aeruginosa was defined as nonsusceptibility to at least one agent in three or more antimicrobial categories according to Magiorakos et al. [22]. Multiple antibiotic resistance index (MARI) was calculated and interpreted for the isolates as described by Gufe et al. [23]. Briefly, it is described as the ratio of the number of antibiotics to which isolates were resistant (a), to the total number of antibiotics to which the isolates were exposed (b), that is, multiple antibiotic resistance index $(\mathrm{MARI})=a / \mathrm{b}$. Bacteria having MARI $>0.2$ originate from high-risk sources of contamination where several antibiotics have been used, while MARI value $\leq 0.2$ indicates strains from sources where antibiotics have seldom or never been used.

2.7. Molecular ESBL and MBL Detection by $r P C R$. Isolated $P$. aeruginosa colonies on Cetrimide agar and CHROMagar Pseudomonas were selected for genomic DNA extraction. Fifteen multidrug isolates were selected from the pool using a simple random sampling technique. Template DNA was extracted by MagNA Pure Compact (MPC) using MPC Nucleic Acid isolation kit according to the manufacturer's instruction. Real-time PCR was carried out in the LightCycler 2.0 instrument (Roche Applied Science, Germany) using Fast start LightCycler 480 HybProbes Master Kit (Roche Diagnostics, USA). Specific primers and probes (Table 3) targeting the genes CTX-M, SHV, TEM, IMP, and VIM were amplified by singleplex rPCR using the same protocol described in Table 2. Primers were designed by TIB Molbiol (Berlin, Germany). rPCR assay was performed in 32 carousels using $20 \mu \mathrm{L}$ capillaries with each capillary containing a total volume of $20 \mu \mathrm{L}$, including $2 \mu \mathrm{L}$ of LightCycler FastStart DNA Master Hybridization Probes (Roche Diagnostics), $2 \mu \mathrm{L}$ of primers $(0.5 \mathrm{mM}$ for each forward and reverse), $2 \mu \mathrm{L}$ of the probe, $2.4 \mu \mathrm{L}$ of $\mathrm{MgCl} 2,2 \mu \mathrm{L}$ of extracted DNA, and water to make up the volume of $20 \mu \mathrm{L}$.

Absolute quantification was carried out using the LightCycler software 4.05. Data were obtained during the annealing period. Fluorescence was measured once every cycle immediately after the $60^{\circ} \mathrm{C}$ incubation (extension step). Fluorescence curves were analyzed with the LightCycler software, version 4.05. Results were expressed by determination of threshold cycle $(\mathrm{Ct})$ value, which signified the cycle at which sample fluorescence became remarkably different from the baseline signal. Positive control strains used included Klebsiella pneumoniae ATCC 51503 (bla $\left.a_{\mathrm{CTX}-\mathrm{M}}\right)$, Klebsiella pneumoniae ATCC 700603 (bla SHV $\left._{\mathrm{S}}\right)$, Escherichia coli NCTC 13351 (bla $\left.a_{\mathrm{TEM}}\right), P$. aeruginosa NCTC 13437 $\left(b l a_{\mathrm{VIM}}\right)$, and Escherichia coli NCTC 13476 (bla $\left.a_{\mathrm{IMP}}\right)$. These were obtained from the National Institute of Communicable Diseases (NICD), Johannesburg, South Africa.

2.8. Statistical Analysis. All the data was entered into an Excel sheet and uploaded onto the SPSS software (version 23.0 IBM, Armonk, NY). The prevalence of multidrug-resistant (MDR) P. aeruginosa and their distribution from different sources (water and abattoir wastewater) were determined and expressed as percentages.

\section{Results}

3.1. Isolation and Antimicrobial Susceptibility Testing. During the period of study, fifty-one isolates of Pseudomonas species were recovered, out of which thirty-six isolates were P. aeruginosa (70.6\%) and fifteen were P. fluorescens/putida (29.4\%). P. aeruginosa was the predominant species, of which nineteen $(52.8 \%)$ and seventeen (47.2\%) originated from surface water and abattoir wastewater, respectively. Of these, the 36 strains of $P$. aeruginosa were selected for further confirmation. They were confirmed by the real-time amplification of the gyrB gene including the reference strain, ATCC 27853 (Figure 1). The results of antibiotic susceptibility testing of $P$. aeruginosa strains showed varying levels of resistance. Of the clinically relevant antibiotics in the panel, there was resistance to aztreonam (86.1\%), ceftazidime (63.9\%), piperacillin (58.3\%), cefepime (55.6\%), imipenem 
Table 1: Primer sequences for detection of gyrB genes.

\begin{tabular}{|c|c|c|c|}
\hline Primers & Primers sequences $\left(5^{\prime}-3^{\prime}\right)$ & $\mathrm{Tm}$ in $0^{\circ} \mathrm{C}$ & References \\
\hline gyr B forward primer & CCT GAC CAT CCG TCG CCA CAA & 55.3 & [19] \\
\hline Gyr B reverse primer & CGC AGC AGG ATG CCG ACG CC & 53.1 & \\
\hline Gyr B probe 1 & FAM-CCG TGG TGG TAG ACC TGT TCC CAG ACC-BHQ & & This study \\
\hline Gyr B probe 2 & FAM-CCG TGG TGG TAG ACC TGT TCC CAG ACC-BBQ & & \\
\hline
\end{tabular}

TABLE 2: rPCR cycle protocol.

\begin{tabular}{lcccc}
\hline Protocol & Temperature $\left({ }^{\circ} \mathrm{C}\right)$ & Acquisition mode & Time & Ramp rate \\
\hline Denaturation & 95 & None & 5 minutes & 4.4 \\
Quantification: Annealing & 95 & None & 30 seconds & 4.4 \\
Extension & 60 & Single & 1 minute & 4.4 \\
Cooling & 40 & None & 30 seconds & 4.4 \\
\hline
\end{tabular}

TABLE 3: Primer sequences for detection of $b l a_{\mathrm{CTX}-\mathrm{M}}, b l a_{\mathrm{SHV}}, b l a_{\mathrm{TEM}}, b l a_{\mathrm{IMP}}$, and $b l a_{\mathrm{VIM}}$ genes.

\begin{tabular}{|c|c|c|c|}
\hline Primers & Primers sequences $\left(5^{\prime}-3^{\prime}\right)$ & $\operatorname{Tm}$ in $0^{\circ} \mathrm{C}$ & References \\
\hline CTX-M forward primer & ATGAGYACCAGTAARGTKATGGC & 58.7 & {$[24]$} \\
\hline CTX-M reverse primer & ATCACKCGGRTCGCCIGGRAT & 59.3 & \\
\hline CTX-M probe & FAM-CCCGACAGCTGGGAGACGAAACGT-BBQ & 70.2 & \\
\hline SHV forward primer & TCCCATGATGAGCACCTTTAAA & 56.8 & {$[25]$} \\
\hline SHV reverse primer & TCCTGCTGGCGATAGTGGAT & 58.6 & \\
\hline SHV probe & FAM-TGCCGGTGACGAACAGCTGGAG-BBQ & 68.3 & \\
\hline TEM forward primer & GCATCTTACGGATGGCATGA & 56.6 & {$[25]$} \\
\hline TEM reverse primer & GTCCTCCGATCGTTGTCAGAA & 57.7 & \\
\hline TEM probe & FAM-CAGTGCTGCCATAACCATGAGTGA-BHQ1 & 62.2 & \\
\hline IMP forward primer & GGGCGGAATAGAGTGGCTTA & 57.6 & {$[26]$} \\
\hline IMP reverse primer & GGCTTGAACCTTACCGTCTTTTT & 59.3 & \\
\hline IMP probe & FAM-CGATCTATCCCCACGTATGCATCTGAATTAACA-BHQ1 & 67.4 & \\
\hline VIM forward primer & TGCGCTTCGGTCCAGTAGA & 59.0 & {$[26]$} \\
\hline VIM reverse primer & TGACGGGACGTATACAACCAGAT & 58.5 & \\
\hline VIM probe & FAM-CTTCTATCCTGGTGCTGCGCATTCG-BHQ1 & 67.6 & \\
\hline
\end{tabular}

(50\%), piperacillin/tazobactam (47.2\%), meropenem (41.7\%), and levofloxacin (30.6\%) (Table 4). Twenty out of thirty-six $P$. aeruginosa strains presented multidrug resistance profiles and were classified as MDR (55.6\%) with 60\% of the MDR strains originating from abattoir wastewater and $40 \%$ being from surface water. Most of the bacteria isolates showed a high MARI ranging from 0.08 to 0.69 with a mean MARI of 0.38 . The mean MARI of isolates from abattoir wastewater was 0.42 while that of aquatic samples equals 0.34 (Figure 2).

3.2. Molecular Detection of Extended Spectrum $\beta$-Lactamase (ESBL) and Metallo- $\beta$-Lactamase (MBL) Encoding Genes in $P$. aeruginosa. PCR screening of genes encoding ESBL and $\mathrm{MBL}$ indicated the amplification of $b l a_{\mathrm{SHV}}, b l a_{\mathrm{CTX}-\mathrm{M}}$, and $b l a_{\text {TEM }}$ in some of the $P$. aeruginosa isolates. Results of molecular detection of ESBL and MBL genotypes in environmental strains of $P$. aeruginosa by rPCR are presented in Table 5. Among the $15 P$. aeruginosa isolates analyzed, 14 isolates $(93.3 \%)$ harbored the $b l a_{\mathrm{SHV}}$, six isolates (40\%) harbored the $b l a_{\mathrm{TEM}}$, and three isolates $(20 \%)$ harbored $b l a_{\text {CTX-M. }}$. Only one isolate $(6.7 \%)$ harbored the $b l a_{\mathrm{VIM}}$ gene while no isolate was detected harboring the MBL, $b l a_{\mathrm{IMP}}$.

\section{Discussion}

In this study, thirty-six isolates of $P$. aeruginosa were recovered from abattoir wastewater and surface water. In agreement with this study, Igbinosa et al. [27-29] have all reported the occurrence of $P$. aeruginosa from hospital drains, environmental, and wastewater networks from various parts of the world. The occurrence of this microorganism is a cause of concern given that it is an opportunistic human pathogen and can infect people whose immunity is compromised [30]. A similar study in Nigeria found that the discharge of effluents from abattoir directly into water bodies without prior treatment has triggered serious health risks subsequent to its contamination by bacteria [14].

The prevalence rate of $P$. aeruginosa (70.6\%) seen in the current study is comparable to previous reports from Nigeria on water samples from fish pond sites and cattle waste, where $P$. aeruginosa was found to be the most prevalent with the highest occurrence rate of $62.8 \%$ and $71.5 \%$, respectively, among other species [31, 32]. This possibly could be due to the physiological versatility and limited nutritional requirements that enable it to adapt in adverse conditions [33]. Likewise, in agreement with this 


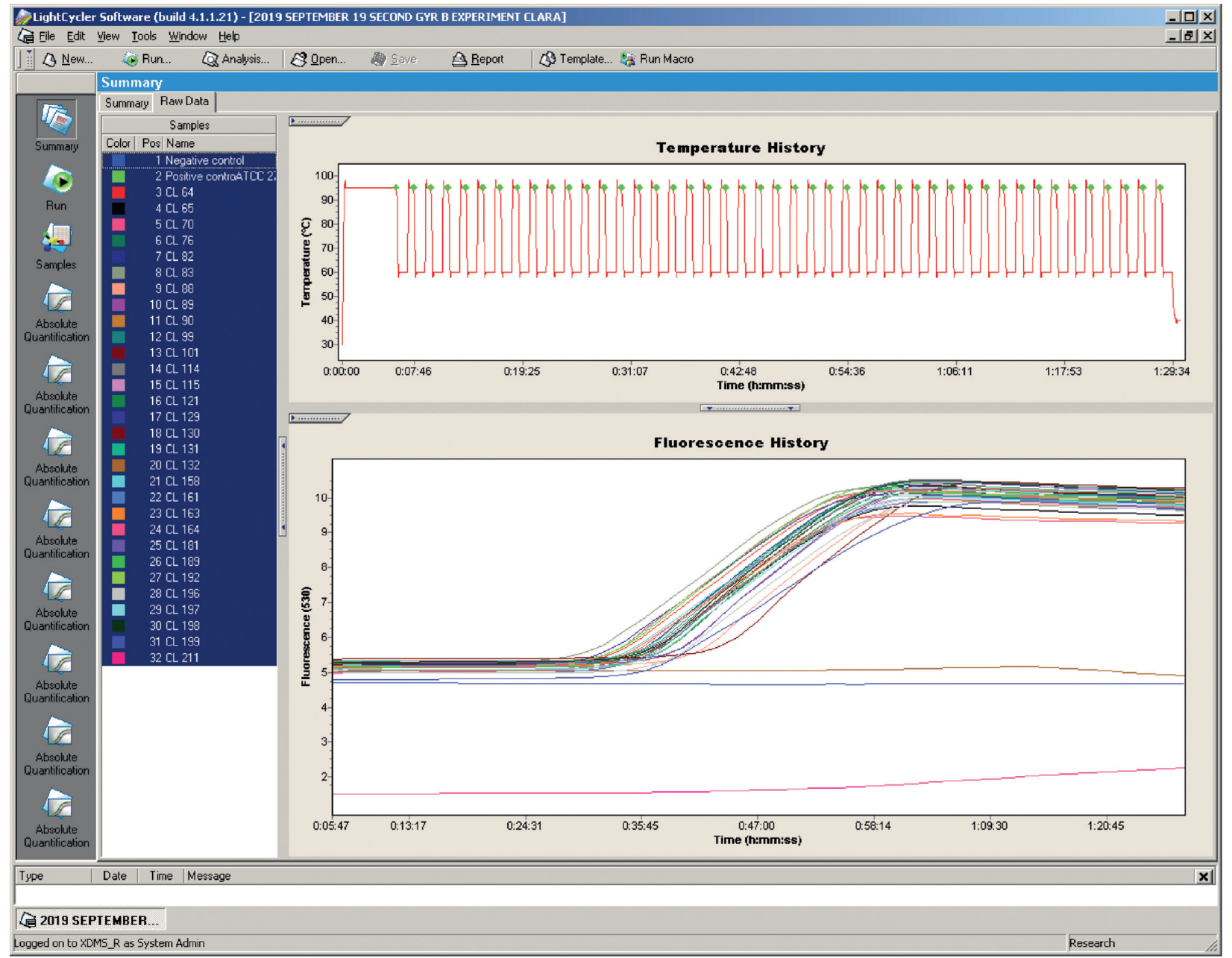

FIgUre 1: Amplification of gyr B (real time with LightCycler 2.0) in P. aeruginosa strains including reference strain ATCC 27853.

TABle 4: Antibiotic resistance pattern of $P$. aeruginosa isolates.

\begin{tabular}{lcc}
\hline Antibiotic & No (\%) resistant & No (\%) susceptible \\
\hline Amikacin & $6(16.7)$ & $30(83.3)$ \\
Aztreonam & $31(86.1)$ & $5(13.9)$ \\
Ceftazidime & $20(55.6)$ & $16(44.4)$ \\
Cefepime & $23(63.9)$ & $13(36.1)$ \\
Ciprofloxacin & $8(22.2)$ & $28(77.8)$ \\
Doripenem & $5(13.9)$ & $31(86.1)$ \\
Gentamicin & $6(16.7)$ & $30(83.3)$ \\
Imipenem & $18(50)$ & $18(50)$ \\
Levofloxacin & $11(30.6)$ & $25(69.4)$ \\
Meropenem & $15(41.7)$ & $21(58.3)$ \\
Piperacillin & $21(58.3)$ & $15(41.7)$ \\
Piperacillin/tazobactam & $17(47.2)$ & $19(52.8)$ \\
Tobramycin & $3(8.3)$ & $33(91.7)$ \\
\hline
\end{tabular}

study, a study carried out in Mafikeng in the North West Province of South Africa isolated P. aeruginosa from both drinking and surface waters [34]. However, contrary to our findings, a study carried out in Alice, Eastern Cape, South Africa, on wastewater samples found a lower occurrence rate of $11.1 \%$ [35]. This disparity is most likely due to different treatment processes used in water purification, or it can be

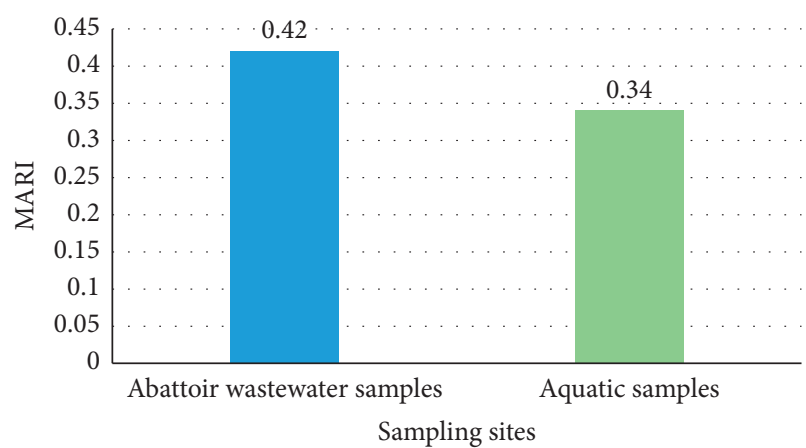

FIgURE 2: Mean MARI values in abattoir wastewater and aquatic $P$. aeruginosa isolates.

assumed that wastewater treatment plant (WWTP) does not totally eliminate bacteria especially MDR strains since these organisms are resilient to the treatment processes and eventually play a role in the transmission and spread of antimicrobial resistance.

The resistance profiles of the isolates revealed $63.9 \%$ and $55.6 \%$ resistance to the second- and third-generation cephalosporin, respectively (ceftazidime and cefepime) and 
TABLE 5: Extended-spectrum $\beta$-lactamase (ESBL) and metallo$\beta$-lactamase (MBL) gene types detected in nonclinical isolates of $P$. aeruginosa.

\begin{tabular}{|c|c|c|}
\hline $\begin{array}{l}\text { Positive by PCR for ESBL } \\
\text { genes }\end{array}$ & $\begin{array}{l}\text { Number amplified } \\
\qquad(N=15)\end{array}$ & $\begin{array}{c}\text { Total } \\
(\%)\end{array}$ \\
\hline \multicolumn{3}{|l|}{ A. Single ESBL gene } \\
\hline$b l a_{\mathrm{SHV}}$ & 14 & 93.3 \\
\hline$b l a_{\mathrm{TEM}}$ & 6 & 40.0 \\
\hline$b l a_{\mathrm{CTX}-\mathrm{M}}$ & 3 & 20.0 \\
\hline$b l a_{\mathrm{VIM}}$ & 1 & 6.7 \\
\hline$b l a_{\mathrm{IMP}}$ & 0 & 0 \\
\hline \multicolumn{3}{|l|}{ B. Two or more ESBL genes } \\
\hline$b l a_{\mathrm{TEM}}+b l a_{\mathrm{SHV}}$ & 6 & 40 \\
\hline$b l a_{\mathrm{TEM}}+b l a_{\mathrm{CTX}-\mathrm{M}}$ & 3 & 20 \\
\hline$b l a_{\mathrm{SHV}}+b l a_{\mathrm{CTX}-\mathrm{M}}$ & 3 & 20 \\
\hline$b l a_{\mathrm{SHV}}+b l a_{\mathrm{VIM}}$ & 1 & 6.7 \\
\hline
\end{tabular}

low resistance to aminoglycosides. The observed resistance pattern in cephalosporins observed in the current study is at par with the previous report of Ejikeugwu et al. [28] but lower compared to that of Tapela et al. $[29,36]$ with a $100 \%$ resistance rate. However, Benie et al. from Cote d'Ivoire [37] reported lower rates of resistance of $6.9 \%$ and $17 \%$ to ceftazidime and cefepime, respectively. A cause for concern is the high resistance displayed to the cephalosporins, which are frontline antipseudomonal drugs for treating $P$. aeruginosa infections; increased resistance to this class of antibiotics will not be favorable and will result in limited treatment options. The present study revealed that $16.7 \%$ of isolates were resistant to aminoglycoside, amikacin, and gentamicin. A similarly low rate of resistance to amikacin (19\%) but slightly higher rate to gentamicin $(28.5 \%)$ was reported by a study carried out in Egypt [36]. However, an elevated resistance rate of $79 \%$ in gentamicin has been reported by a study carried out in Nigeria [28]. This variation could be due to differences in the prescription pattern of aminoglycoside antibiotics [38].

Resistance to carbapenems including imipenem (50\%) and meropenem (41.7\%) was also observed in the current study. This is quite unexpected, given the fact that carbapenems represent one of the most effective and among the best options for treating Gram-negative infections particularly MDR infections. Carbapenem-resistant $P$. aeruginosa isolates are frequently associated with a higher mortality rate due to the enzyme carbapenemase mediating the resistance and a higher likelihood of extensive spread of resistance through mobile genetic elements [39]. The abuse of antimicrobials in human and veterinary medicine often leads to a proliferation of antibiotic-resistant bacteria (ARB) and antibiotic-resistant genes (ARGs) that can be transferred to human pathogenic bacteria. This transfer eventually nullifies the efficacy of current and upcoming antibiotics, thereby leading to treatment failure for some life-threatening diseases [40].

In the current study, the prevalence of $55.6 \%$ MDR $P$. aeruginosa (MDRPA) in a nonclinical setting is high and alarming. Consequently, this study proved the presence of MDR $P$. aeruginosa in the nonclinical environment in the
Eastern Cape Province of South Africa. This finding is consistent with the report of Algammal et al. [41] with 55.5\% MDR strains of $P$. aeruginosa from freshwater fish samples. However, Olga et al. [42] reported a lower rate of MDRPA of $32 \%$ from the aquatic environment. The irrational and unwarranted use of antibiotics fast-tracks the increase of multidrug-resistant strains, thus rendering empirical antibiotic therapy ineffective [43].

MAR indexing method is a simplified rapid method of distinguishing organisms from different origins either from high-risk sources of contamination where antibiotics are frequently used or from low-risk sources [44] and as an indicator of the level of contamination potentially unsafe for humans [45]. A MAR index $>0.2$ indicates that isolates originate from high-risk sources of contamination [46]. In the current study, the analysis of the MAR index of the $P$. aeruginosa strains showed that all of them had a MAR index above 0.2 (Figure 2). Odjajare et al. [47] and Gufe et al. [23] reported similar results. The findings reflect the overuse of antibiotics in animal production and highlight the sources of these pathogens, which eventually translocate into water bodies and pose health risks to humans.

The release of MDR bacteria including ESBL and MBL producers into water bodies is a cause of concern. These organisms could act as opportunistic pathogens when they persist in the environment, and since they carry mobile genetic material, they can serve as a resistance pool that could fast-track the spread of antimicrobial resistance [48]. In the current study, bla $a_{\mathrm{SHV}}$ was the most prevalent ESBL detected by PCR. This was detected in 14 isolates (93.3\%). $b l a_{\text {TEM }}$ was detected in $40 \%$ of the isolates while the least detected ESBL was $b l a_{\text {CTX-M }}(20 \%)$. This study is in agreement with other authors $[49,50]$. Together, these statistics suggest successful dissemination of the ESBLencoding genes universally.

4.1. Limitations. The limitation of the study is that the study was based on a small sampling size.

\section{Conclusions}

The findings of this study revealed a considerable burden of resistance against important antibiotics such as ceftazidime, cefepime, imipenem, and meropenem including piperacillin and piperacillin/tazobactam, which are antibiotics of choice for treating MDR P. aeruginosa. This poses complications to the successful treatment of human infections. Given the public health relevance, the results of this study reveal the importance and necessity of concerted surveillance of antimicrobial resistance and resistance genes in the nonclinical environment at both local and regional levels and the implementation of the One Health approach. In addition, the occurrence of ESBL-producing P. aeruginosa presents a potential public health threat since the genetic elements responsible for this resistance are present on mobile genetic elements (MGEs) that can be transferred to other Gramnegative bacteria through horizontal gene transfer. 


\section{Data Availability}

All data generated or analyzed during this study are included in this published article.

\section{Ethical Approval}

This study was approved by the Human Research Ethics Committee of the Faculty of Health Sciences of Walter Sisulu University approved this study (Reference number: 024/ 2016).

\section{Disclosure}

The funders had no role in study design, data collection, analysis and interpretation of data, writing of the manuscript, and decision to publish.

\section{Conflicts of Interest}

The authors declare that there are no conflicts of interest regarding the publication of this paper.

\section{Authors' Contributions}

Apalata T., Vasaikar S., and Hosu M. C. conceived and designed the experiment. Hosu M. C. and Okuthe G. E. were involved in the field and experimental studies. Hosu M. C., Okuthe G. E., and Apalata T. collected the data, analyzed the data, and drafted the manuscript. Apalata T., Vasaikar S., Hosu M. C., and Okuthe G. E. revised the manuscript critically for intellectual content. All authors read and approved the final manuscript.

\section{Acknowledgments}

The authors are grateful to the Nelson Mandela Academic Hospital (NMAH) and the Microbiology Laboratory at the National Health Laboratory Services (NHLS) at the NMAH for support during sample collection. The National Institute of Communicable Diseases (NICD) is appreciated for the supply of reference strains. Hosu M. C received a Ph.D. bursary from the National Research Foundation (NRF) in this study. The study was conducted within the Thuthuka Rating Track (Ref. No. TTK150625121238; UID: 99307) project funded by NRF (South African).

\section{References}

[1] S. Allcock, E. H. Young, M. Holmes et al., "Antimicrobial resistance in human populations: challenges and opportunities-ERRATUM," Global Health, Epidemiology and Genomics, vol. 2, 2017.

[2] S. M. Cahill, P. Desmarchelier, V. Fattori, A. Bruno, and A. Cannavan, "Global perspectives on antimicrobial resistance in the food chain," Food Protection Trends, vol. 37, no. 5, pp. 353-360, 2017.

[3] K. Hoelzer, N. Wong, J. Thomas, K. Talkington, E. Jungman, and A. Coukell, "Antimicrobial drug use in food-producing animals and associated human health risks: what, and how strong, is the evidence?" BMC Veterinary Research, vol. 13, p. 211, 2017.

[4] A. Yaw Anane, T. Apalata, S. Vasaikar, G. E. Okuthe, and S. Songca, "Prevalence and molecular analysis of multidrugresistant Acinetobacter baumannii in the extra-hospital environment in Mthatha, South Africa," Brazilian Journal of Infectious Diseases, vol. 23, no. 6, pp. 371-380, 2019.

[5] B. Malik and B. Samit, "Antibiotic drug-resistance as a complex system driven by socio-economic growth and antibiotic misuse," Scientific Reports, vol. 9, p. 9788, 2019.

[6] E. K. Rousham, L. Unicomb, and M. A. Islam, "Human, animal and environmental contributors to antibiotic resistance in low-resource settings: integrating behavioural, epidemiological and One Health approaches," Proceedings of the Royal Society B: Biological Sciences, vol. 285, no. 1876, p. 20180332, 2018.

[7] I. T. Carvalho and L. Santos, "Antibiotics in the aquatic environments: a review of the European scenario," Environment International, vol. 94, pp. 736-757, 2016.

[8] A. Deredjian, C. Colinon, E. Hien et al., "Low occurrence of Pseudomonas aeruginosa in agricultural soils with and without organic amendment," Frontiers in Cellular and Infection Microbiology, vol. 4, p. 53, 2014.

[9] E. A. Elshafiee, S. M. Nader, S. M. Dorgham, and D. A. Hamza, "Carbapenem-resistant Pseudomonas aeruginosa originating from farm animals and people in Egypt," Journal of Veterinary Research, vol. 63, no. 3, pp. 333-337, 2019.

[10] A. Golle, S. Janezic, and M. Rupnik, "Low overlap between carbapenem resistant Pseudomonas aeruginosa genotypes isolated from hospitalized patients and wastewater treatment plants," PLoS One, vol. 12, no. 10, 2017.

[11] E. Peterson and P. Kaur, "Antibiotic resistance mechanisms in bacteria: relationships between resistance determinants of antibiotic producers, environmental bacteria, and clinical pathogens," Frontiers in Microbiology, vol. 9, p. $2928,2018$.

[12] H. Heuer, H. Schmitt, and K. Smalla, "Antibiotic resistance gene spread due to manure application on agricultural fields," Current Opinion in Microbiology, vol. 14, no. 3, pp. 236-243, 2011.

[13] E. I. Atuanya, N. A. Nwogu, and C. U. Orah, "Antibiotic resistance and plasmid profiles of bacteria isolated from abattoir effluents around Ikpoba river in Benin city, Nigeria," Journal of Applied Sciences \& Environmental Management, vol. 22, no. 11, pp. 1749-1755, 2018.

[14] I. R. Iroha, O. B. Eromonsele, I. B. Moses, F. N. Afiukwa, A. E. Nwakaeze, and P. C. Ejikeugwu, "In vitro antibiogram of multidrug resistant bacteria isolated from Ogbete abattoir effluent in Enugu State, Nigeria," International Journal of Environmental Research and Public Health, vol. 3, no. 1, 2016.

[15] A. E. Laudy, P. Róg, K. Smolińska-Król et al., "Prevalence of ESBL-producing Pseudomonas aeruginosa isolates in Warsaw, Poland, detected by various phenotypic and genotypic methods," PLoS One, vol. 12, no. 6, 2017.

[16] S. Dehbashi, H. Tahmasebi, M. Y. Alikhani, F. Keramat, and M. R. Arabestani, "Distribution of Class B and Class A $\beta$-lactamases in clinical strains of Pseudomonas aeruginosa: comparison of phenotypic methods and high-resolution melting analysis (HRMA) assay," Infection and Drug Resistance, vol. 13, pp. 2037-2052, 2020.

[17] American Public Health Association (APHA), Standard Methods for the Examination of Water and Wastewater, APHA, Washington, DC, USA, 22nd edition, 2012. 
[18] M. Goudarzi, M. Fazeli, M. Azad, S. S. Seyedjavadi, and R. Mousavi, "Aloe vera gel: effective therapeutic agent against multidrug-resistant Pseudomonas aeruginosa isolates recovered from burn wound infections," Chemotherapy Research and Practice, vol. 2015, Article ID 639806, 5 pages, 2015.

[19] S. N. Anuj, D. M. Whiley, T. J. Kidd et al., "Identification of Pseudomonas aeruginosa by a duplex real-time polymerase chain reaction assay targeting the ecfX and the gyrB genes," Diagnostic Microbiology and Infectious Disease, vol. 63, no. 2, pp. 127-131, 2009.

[20] J. D. Lutgring, A. Kim, D. Campbell, M. Karlsson, A. C. Brown, and E. M. Burd, "Evaluation of the MicroScan colistin well and gradient diffusion strips for colistin susceptibility testing in Enterobacteriaceae," Journal of Clinical Microbiology, vol. 57, no. 5, 2019.

[21] Clinical and Laboratory Standards Institute (CLSI), Performance Standards for Antimicrobial Susceptibility Testing; 20th Informational Supplement. CLSI Document M100-S20, 2010, Clinical and Laboratory Standards Institute, Wayne, PA, USA.

[22] A. P. Magiorakos, A. Srinivasan, R. B. Carey et al., "Multidrugresistant, extensively drug-resistant and pandrug-resistant bacteria: an international expert proposal for interim standard definitions for acquired resistance," Clinical Microbiology and Infection, vol. 18, no. 3, pp. 268-281, 2012.

[23] C. Gufe, T. Canaan Hodobo, B. Mbonjani et al., "Antimicrobial profiling of bacteria isolated from fish sold at informal market in Mufakose, Zimbabwe," International Journal of Microbiology, vol. 2019, Article ID 8759636, 7 pages, 2019.

[24] S. Angeletti, G. Gherardi, L. De Florio et al., "Real-time polymerase chain reaction with melting analysis of positive blood culture specimens in bloodstream infections: diagnostic value and turnaround time," New Microbiologica, vol. 36, pp. 65-74, 2013.

[25] N. Roschanski, J. Fischer, B. Guerra, and U. Roesler, "Development of a multiplex real-time PCR for the rapid detection of the predominant beta-lactamase genes CTX-M, SHV, TEM and CIT-type AmpCs in Enterobacteriaceae," PLoS One, vol. 9, no. 7, 2014.

[26] A. F. Wendel, A. H. Brodner, S. Wydra et al., "Genetic characterization and emergence of the metallo- $\beta$-lactamase GIM-1 in Pseudomonas spp. and Enterobacteriaceae during a long-term outbreak," Antimicrobial Agents and Chemother$a p y$, vol. 57, no. 10, pp. 5162-5165, 2013.

[27] I. H. Igbinosa, A. Beshiru, and E. O. Igbinosa, "Antibiotic resistance profile of Pseudomonas aeruginosa isolated from aquaculture and abattoir environments in urban communities," Antimicrobial Agents and Chemotherapy, vol. 7, no. 1, pp. 47-52, 2017.

[28] C. Ejikeugwu, C. Esimone, I. Iroha, P. Eze, M. Ugwu, and M. Adikwu, "Genotypic and phenotypic characterization of MBL genes in Pseudomonas aeruginosa isolates from the nonhospital environment," Journal of Pure and Applied Microbiology, vol. 12, no. 4, pp. 1877-1885, 2018.

[29] K. Tapela and T. Rahube, "Isolation and antibiotic resistance profiles of bacteria from influent, effluent and downstream: a study in Botswana," African Journal of Microbiology Research, vol. 13 , no. 15 , pp. 279-289, 2019.

[30] S. C. Onuoha, "Distribution and antibiogram of bacterial species in effluents from abattoirs in Nigeria," Journal of Environmental and Occupational Science, vol. 7, no. 1, pp. 1-8, 2018.

[31] O. I. Falodun and E. O. Ikusika, "Extended spectrum betalactamase and metallo beta-lactamase producing Pseudomonas species isolated from fish pond water in Ibadan, Nigeria,"
International Journal of Environmental Studies, vol. 77, no. 3, 2019.

[32] O. I. Falodun and I. B. Musa, "Pseudomonas Species from cattle dung producing extended spectrum and metallo-beta lactamases," European Journal of Biological Research, vol. 10, no. 1, pp. 1-10, 2020.

[33] M. F. Moradali, S. Ghods, and B. H. Rehm, "Pseudomonas aeruginosa lifestyle: a paradigm for adaptation, survival, and persistence," Frontiers in Cellular and Infection Microbiology, vol. 7, p. 39, 2017.

[34] S. G. Mulamattathil, C. Bezuidenhout, M. Mbewe, and C. N. Ateba, "Isolation of environmental bacteria from surface and drinking water in Mafikeng, South Africa, and characterization using their antibiotic resistance profiles," Journal of Pathogens, vol. 2014, Article ID 371208, 11 pages, 2014.

[35] I. H. Igbinosa, U. U. Nwodo, A. Sosa, M. Tom, and A. I. Okoh, "Commensal Pseudomonas species isolated from wastewater and freshwater milieus in the Eastern Cape Province, South Africa, as reservoir of antibiotic resistant determinants," International Journal of Environmental Research and Public Health, vol. 9, pp. 2537-2549, 2012.

[36] M. Elhariri, D. Hamza, R. Elhelw, and S. M. Dorgham, "Extended-spectrum beta-lactamase-producing Pseudomonas aeruginosa in camel in Egypt: potential human hazard," Annals of Clinical Microbiology and Antimicrobials, vol. 16, no. 1, p. 21, 2017.

[37] C. K. D. Benie, G. Nathalie, D. Adjéhi et al., "Prevalence and antibiotic resistance of Pseudomonas aeruginosa isolated from bovine meat, fresh fish and smoked fish," Archives of Clinical Microbiology, vol. 8, no. 3, 2017.

[38] A. H. Asghar and O. B. Ahmed, "Prevalence of aminoglycoside resistance genes in Pseudomonas aeruginosa isolated from a tertiary care hospital in Makkah, KSA," Clinical Practice, vol. 15, no. 2, pp. 541-547, 2018.

[39] A. C. Juayang, J. P. Lim, A. F. Bonifacio et al., "Five-year antimicrobial susceptibility of Pseudomonas aeruginosa from a local tertiary hospital in Bacolod City, Philippines," Tropical Medicine and Infectious Disease, vol. 2, no. 3, p. 28, 2017.

[40] A. Goulas, B. Livoreil, N. Grall et al., "What are the effective solutions to control the dissemination of antibiotic resistance in the environment? A systematic review protocol," Environmental Evidence, vol. 7, no. 1, 2018.

[41] A. M. Algammal, M. Mabrok, E. Sivaramasamy et al., "Emerging MDR-Pseudomonas aeruginosa in fish commonly harbor opr L and tox A virulence genes and bla TEM, bla CTX-M, and tet A antibiotic-resistance genes," Scientific Reports, vol. 10, p. 15961, 2020.

[42] P. Olga, V. Apostolos, G. Alexis, V. George, and M. Athena, "Antibiotic resistance profiles of Pseudomonas aeruginosa isolated from various Greek aquatic environments," FEMS Microbiology Ecology, vol. 92, no. 5, 2016.

[43] Z. Pang, R. Raudonis, B. R. Glick, T. J. Lin, and Z. Cheng, "Antibiotic resistance in Pseudomonas aeruginosa: mechanisms and alternative therapeutic strategies," Biotechnology Advances, vol. 37, no. 1, pp. 177-192, 2019.

[44] Z. Nain, M. Islam, and M. Minnatul Karim, "Antibiotic resistance profiling and molecular phylogeny of biofilm forming bacteria from clinical and non-clinical environment in southern part of Bangladesh," International Journal of Enteric Pathogens, vol. 7, no. 2, pp. 37-43, 2019.

[45] M. M. Kathleen, L. Samuel, C. Felecia et al., "Antibiotic resistance of diverse bacteria from aquaculture in Borneo," International Journal of Medical Microbiology, vol. 2016, Article ID 2164761, 9 pages, 2016. 
[46] T. A. Stenström, A. I. Okoh, and A. A. Adegoke, "Antibiogram of environmental isolates of Acinetobacter calcoaceticus from nkonkobe municipality, South Africa," Fresenius Environmental Bulleti, vol. 25, no. 8, pp. 3059-3065, 2016.

[47] E. E. Odjadjare, E. O. Igbinosa, R. Mordi, B. Igere, C. L. Igeleke, and A. I. Okoh, "Prevalence of multiple antibiotics resistant (MAR) Pseudomonas species in the final effluents of three municipal wastewater treatment facilities in South Africa," International Journal of Environmental Research and Public Health, vol. 9, no. 6, pp. 2092-2107, 2012.

[48] C. C. Miranda, I. de Filippis, L. H. Pinto et al., "Genotypic characteristics of multidrug-resistant Pseudomonas aeruginosa from hospital wastewater treatment plant in Rio de Janeiro, Brazil," Journal of Applied Microbiology, vol. 118, no. 6, pp. 1276-1286, 2015.

[49] C. I. Chikwendu, S. N. Ibe, and G. C. Okpokwasili, "Detection of blaSHV and blaTEM beta-lactamase genes in multi-resistant Pseudomonas isolates from environmental sources," African Journal of Microbiology Research, vol. 5, no. 15, pp. 2067-2074, 2011.

[50] L. S. Tew, L. Y. She, and C. H. Chew, "Isolation, antimicrobial susceptibility profile and detection of Sull, blaTEM, and blaSHV in amoxicillin-clavulanate-resistant bacteria isolated from retail sausages in Kampar, Malaysia," Jundishapur Journal of Microbiology, vol. 9, no. 10, Article ID e37897, 2016. 